\title{
Diseño decorativo de pavimentos cerámicos adaptado a inyección de tinta mediante tratamiento digital de imagen
}

\author{
B. DEFEZ*, V. SANTIAGO-PRADERAS, E. LLUNA, G. PERIS-FAJARNÉS, L. DUNAI \\ Centro de Investigación en Tecnologías Gráficas, Universitat Politècnica de València \\ Camino de Vera s/n-8H, 46022 Valencia, España \\ bdefez@degi.upv.es
}

\begin{abstract}
El sector productivo de pavimentos y revestimientos cerámicos es una industria muy competitiva. La capacidad de los diseñadores de ofrecer modelos con nuevos diseños de la cara vista, adaptados a los medios de producción, juega un papel muy importante en la competitividad. En el presente trabajo se analiza la evolución del proceso de diseño en el sector cerámico, así como los cambios experimentados de forma paralela por los medios de impresión. A continuación se presenta un nuevo concepto de diseño de baldosa cerámica, basado en procesado de imagen digital. Esta técnica permite la generación de diseños homogéneos y no repetitivos de grandes superficies, especialmente pensados para la decoración mediante inyección de tinta. Con los algoritmos programados se ha creado un programa informático prototipo de ayuda al diseño cerámico. Esta herramienta permite crear diseños continuos para grandes superficies ahorrando tiempo de desarrollo.
\end{abstract}

Palabras clave: pavimentos cerámicos, procesado de imagen digital, impresión inkjet.

Decorative design of ceramic tiles adapted to inkjet printing employing digital image processing

The ceramic tile sector is a very competitive industry. The designer's proficiency to offer new models of the decorated surface, adapted to the production means, plays a very important role in the competitiveness. In the present work, we analyze the evolution of the design process in the ceramic sector, as much as the changes experimented in parallel by the printing equipment. Afterwards, we present a new concept of ceramic design, based on digital image processing. This technique allows the generation of homogeneous and non-repetitive designs for large surfaces, especially thought for inkjet printing. With the programmed algorithms we have compiled a prototype software for the assistance of the ceramic design. This tool allows creating continuous designs for large surfaces saving developing time.

Keywords: ceramic tile, digital image processing, inkjet printing.

\section{INTRODUCCIÓN}

\subsection{Diseño y decoración cerámica actual}

El sector productivo de baldosas cerámicas se enfrenta a un mercado muy competitivo, con un consumidor final muy exigente. Los investigadores junto con las empresas han apostado, especialmente, por la mejora de los materiales cerámicos, y la diversificación de los diseños de la cara vista.

En relación al diseño decorativo, los creativos cerámicos ofrecen año tras año nuevas propuestas. Se combinan los motivos más tradicionales con estéticas innovadoras, como la imitación a madera, metal, o piedra natural; o el uso de relieves o paisajes como inspiración. Sin embargo, el proceso de diseño en sí mismo apenas ha evolucionado. Muchas empresas han integrado herramientas CAD (Computer Aided Design) en sus departamentos, pero su uso continúa siendo muy artesanal. En la mayoría de los casos, se toman imágenes de partida, directamente adquiridas por la empresa o compradas a diseñadores externos, y se retocan mediante programas comerciales de edición de imagen digital (como
Photoshop de Adobe) para conseguir determinados efectos y adaptar la imagen a la tecnología de decoración. Los equipos tradicionales de impresión son esencialmente máquinas de impresión rotatoria mediante huecograbado y pantallas serigráficas. Mediante ambas tecnologías, la decoración de la baldosa se produce por la deposición secuencial de las distintas tintas. Es decir, si un diseño está compuesto por tres colores distintos, la pieza pasará por tres pantallas serigráficas o tres rodillos de hueco grabado de forma secuencial para recibir la decoración final. Este proceso de diseño puede ser adecuado para decoraciones sencillas (planas o lisas), cuyos colores se obtengan mediante la combinación de un número reducido de tintas (típicamente tres tintas). En cambio, para decoraciones con motivos o texturas complejas, donde además se buscan colores intermedios difíciles de obtener mediante combinaciones simples de tintas, las tecnologías citadas no son adecuadas: su precisión y variedad de decoración son insuficientes. Además, estos sistemas tradicionales tienen una alta variabilidad (diferencias de color no deseadas entre baldosas), lo que da lugar a porcentajes altos de producto 
no conforme y costes añadidos de producción [1]. Al mismo tiempo, son sistemas de gran inercia productiva, por lo que deben ser empleados con lotes de producción grandes, algo cada vez más en desuso en la industria.

La combinación de estos dos factores (el de diseño y el tecnológico) había dado lugar a un proceso de diseño cerámico lento y rígido. Todo ello ha redundado en costes innecesarios de lanzamiento, ciclos más largos de desarrollo y fabricación, y stocks a veces insufribles. Este malgasto de tiempo y dinero era totalmente inadmisible en una industria tan competitiva, además de restringir enormemente las posibilidades creativas y organizativas.

\subsection{Nueva tecnología de decoración cerámica por inyección de tinta}

La decoración de superficies mediante inyección de tinta, conocida también como decoración por chorro de tinta o simplemente "ink-jet" o "inkjet", permite la impresión de decoraciones complejas multicolor de alta calidad. Mediante esta tecnología, los colores finales son generados sobre la superficie a decorar mediante la deposición muy cercana de pequeñas gotas de tintas distintas de un grupo de tintas que comúnmente incluye cian, magenta, amarillo y negro (espacio de color CMYK). El ojo humano percibe este fino tramado de gotas de distintos colores básicos como superficies homogéneas del color mezcla o intermedio. Así mismo, se pueden imprimir motivos y texturas de todo tipo a alta resolución, sin las limitaciones técnicas de las tecnologías tradicionales.

Cabe destacar que la decoración se produce sin contacto con la pieza, permitiendo decorar relieves más pronunciados. Además, es posible decorar el $100 \%$ de la superficie. Por otro lado, tratándose de una tecnología digital, el diseño pasa de forma directa del ordenador a la máquina, por lo que es más sencillo realizar pruebas o modificaciones en el diseño, consiguiendo un proceso productivo más flexible.

La decoración inkjet ha sido ampliamente usada en el campo de las artes gráficas desde sus primeros desarrollos en la década de los 90. Sin embargo, su entrada en el sector cerámico es mucho más reciente. Esto se ha debido, entre otros factores, a la necesidad de desarrollar tintas aptas para la inyección, que respondieran adecuadamente a las altas temperaturas alcanzadas durante el proceso de cocción.

Actualmente, la decoración de baldosa cerámica mediante impresoras de inyección de tinta es una tecnología en expansión que se está consolidando rápidamente, y que puede cubrir las carencias del huecograbado y la serigrafía. Las principales multinacionales cerámicas ya han integrado o se encuentran en fase de integración de esta tecnología [2-6]. Una vez implementada, la tecnología debe explotarse en toda su magnitud, para ello, es necesario reformar también el proceso de diseño mediante mecanismos asistidos por computador que permitan agilizar y flexibilizar la producción de decoraciones innovadoras de alta calidad.

Con todo ello, la introducción de procesado digital de imagen en el diseño cerámico, así como el uso de la inyección de tinta para la impresión, pueden constituir una alternativa para solucionar los inconvenientes de los métodos y tecnologías tradicionales.

\subsection{Uso de procesado digital de imagen en el proceso productivo cerámico}

El procesado digital de imagen es una técnica con escasa presencia en el proceso de producción de la baldosa cerámica. Esencialmente, se ha usado en la fase de clasificación para la detección de defectos superficiales y cambios de color (o tono). Los trabajos más destacados en el ámbito de la clasificación automática han sido desarrollados en Asian University of Bangladesh, The University of New South Wales, University of Science and Technology (Corea) y University of Surrey (Reino Unido) [7-10]. En el campo de la predicción de color, los trabajos más relevantes han tenido lugar en la Universidad Politécnica de Valencia, Università di Modena e Reggio Emilia (Italia), University of Joensuu (Finlandia) y University of Derby (Reino Unido) [11-14].

En relación al proceso de diseño de la cara vista del producto, realizado previamente a fabricación, el procesado de imagen apenas ha sido usado. Algunos investigadores han tratado de ofrecer herramientas de ayuda a la gestión del proceso de diseño en sí mismo basado en este procesado [15-17].

El uso de procesamiento de imagen digital para la adaptación del proceso de diseño de decoraciones cerámicas a la impresión por inyección de tinta es un campo de investigación nuevo. Como se citó en el apartado anterior, la mayoría de las empresas que ya emplean o están empezando a emplear la decoración inkjet, adaptan sus diseños mediante el uso de aplicaciones comerciales de tratamiento deimagen.Sinembargo, estas aplicaciones únicamente permiten transformaciones automáticas simples. La obtención de imágenes adaptadas, listas para imprimir, del tamaño, resolución, textura y color deseado recae en la pericia completamente artesanal del diseñador. Es el diseñador quien debe generar una imagen que represente una textura homogénea para un gran formato, a partir de una pequeña muestra de la textura. La forma en que se consigue la imagen de gran formato depende completamente de la estrategia particular de cada diseñador: replicar o aumentar la imagen inicial, combinar varias imágenes diferentes de texturas similares, incluso crear parte de la imagen desde cero empleando un programa de dibujo artístico, etc. A continuación, el diseñador debe revisar la superficie final al completo, en toda su extensión, y detectar las áreas en donde el diseño pierde homogeneidad, para retocarlo con las herramientas disponibles de nuevo en base a su propia experiencia. Algunas prácticas típicas del retoque de imágenes incluyen: cambiar manualmente el color de determinados píxeles, rotar o deformar pequeñas áreas de la imagen, añadir parches de otras imágenes que después se vuelven a retocar en sus contornos para que parezcan parte de la textura homogénea, etc.

\section{OBJETIVO DEL TRABAJO}

El objetivo de esta investigación es mejorar el proceso de diseño de imágenes que representen texturas homogéneas para la impresión de grandes superficies cerámicas.

Para conseguir este objetivo, se ha definido y desarrollado una herramienta informática de asistencia al diseño cerámico, que permita la creación de estas imágenes en un tiempo y a un coste inferior al consumido mediante las prácticas tradicionales de diseño. 
En este trabajo se exponen distintas técnicas de procesado de imagen digital para automatizar la creación de nuevos diseños especialmente pensados para su impresión mediante inyección de tinta. En base a estas técnicas se han desarrollado códigos propios de procesado y se ha generado el programa prototipo. Mediante la "automatización" no se limita en absoluto el trabajo del diseñador sino todo lo contrario. Se trata de dotarlo de herramientas computacionales que le permitan centrarse por completo en la estética de su diseño, de manera que la adaptación del mismo a las características de un determinado producto (tamaño, necesidades de resolución, etc.) sea resuelta con la ayuda del computador. Se pretende potenciar la diversidad y creatividad de estos diseños, flexibilizar y automatizar el proceso de diseño en sí mismo, y optimizar la decoración en la línea de fabricación, de forma que el resultado final del proceso se corresponda con el diseño inicial de forma óptima.

Se busca integrar el nuevo concepto de diseño cerámico, basado en procesado de imagen digital automatizado, con la nueva tecnología de impresión cerámica por medio de inyección de tinta. Esta combinación permitirá crear y gestionar diseños cerámicos en un abanico mucho más amplio, con una inversión de tiempo y dinero inferior.

\begin{tabular}{|c|c|c|}
\hline \multirow{14}{*}{$\begin{array}{l}\text { PATTERNS } \\
\text { LIBRARY }\end{array}$} & $\begin{array}{l}\text { OUTPUT IMAGE } \\
\text { REQUIREMENTS }\end{array}$ & \multirow{14}{*}{$\begin{array}{l}\text { INPUT } \\
\text { IMAGE }\end{array}$} \\
\hline & BACKGROUND BUILDING & \\
\hline & EMPTY BACKGROUND & \\
\hline & SYMMETRIC REPLICATION & \\
\hline & BACKGROUND & \\
\hline & PATTERNS SELECTION & \\
\hline & + & \\
\hline & $\begin{array}{c}\text { PATTERNS COORDINATES } \\
\text { EXTRACTION }\end{array}$ & \\
\hline &  & \\
\hline & $\begin{array}{l}\text { TEMPORAL VARIABLES } \\
\text { INSERTION }\end{array}$ & \\
\hline & OUTPUT IMAGE & \\
\hline & INKJET PRINTER & \\
\hline & 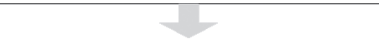 & \\
\hline & CERAMIC TILE LOT & \\
\hline
\end{tabular}

Fig. 1. Esquema del programa desarrollado

En la Fig. 1 puede observarse el esquema básico de la herramienta y su relación con el posterior proceso de decoración cerámico.

\section{PROGRAMA DESARROLLADO}

\subsection{Bases del procesamiento digital de imagen}

El procesamiento digital de imagen se fundamenta en el diseño y posterior programación de algoritmos matemáticos sobre soportes informáticos. Los algoritmos manipulan una imagen de partida (diseño o boceto inicial), tratándola como una matriz de datos, en donde cada entrada de la matriz contiene las coordenadas de color del píxel de la imagen situado en esa posición. La manipulación da lugar a una imagen de salida, de características diferentes y deseadas, que será imprimida en la baldosa mediante la tecnología existente. Existen tres modelos de procesamiento generales: espacial, en el dominio de la frecuencia y básico [18].

En primer lugar, el procesamiento espacial está formado por aquellas técnicas que operan directamente sobre los valores de los píxeles de la imagen. Admite las operaciones aritméticas más usadas en procesamiento de imágenes (suma, resta, multiplicación y división), así como las operaciones lógicas esenciales (and , or , not) siendo necesario que ambas imágenes tengan el mismo tamaño para poder ser llevadas a cabo. Además permite diversas transformaciones geométricas que modifican las relaciones espaciales entre píxeles. Las más importantes son: interpolación (estimación de los valores de una imagen en una sección específica); amplificación y reducción de imágenes; convolución bidimensional discreta (base de algunos procesamientos comunes como el filtrado de imágenes); correlación (utilizada para encontrar el parecido existente entre píxeles de una imagen); y traslación (desplazamiento físico de alguna sección que forma parte de la imagen).

En segundo lugar, el proceso en el dominio de la frecuencia es aquel en el que una imagen se representa como la suma de señales periódicas con diferentes frecuencias. La manera de manifestarse es a través de dos tipos de transformadas: la de Fourier, que es la expresión de una función periódica (series de Fourier) o no periódicas (transformada de Fourier) en el dominio del tiempo; y la discreta del coseno, que representa una imagen como la suma de funciones senoidales de diferentes amplitudes y frecuencias. Cabe destacar que mediante estas transformadas es posible obtener aplicaciones prácticas sobre el tratamiento de la imagen, como por ejemplo una respuesta a la frecuencia de filtros lineales, la convolución rápida, la localización de características en imágenes o la amplificación de las mismas.

Por último, el procesamiento de imágenes básico, tal y como su nombre indica, acepta la ejecución de ciertos procesos generales como son: binarización (comparación de los niveles de gris presentes en la imagen con un valor (umbral) predeterminado para su clasificación); filtrado (recalculo del valor de determinados píxeles en función de los píxeles vecinos los cuales permiten realizar el tratamiento del ruido en las imágenes); realce de bordes (resalte de aquellos píxeles que tienen un valor de gris diferente al de sus vecinos y que se interpretan como el contorno de una forma); detección de contornos; manipulación del contraste; modificación del histograma; y técnicas basadas en el gradiente [19-21].

\subsection{Desarrollo de la programación para decoración cerámica}

En el programa desarrollado, la entrada al sistema es una imagen inicial. Se trata de una muestra de tamaño reducido (entre 60 y $100 \mathrm{~cm}^{2}$ ) y resolución media (entre 180 y 300 píxeles por pulgada) de una textura aleatoria que debe ser imprimida en un lote cerámico de superficie muy superior a la de la imagen inicial. La imagen puede presentar un amplio grupo 
de formatos, como Tagged Image File Format (tiff), Joint Photographic Experts Group (jpeg ó jpg), Portable Network Graphics (png), Graphic Interchange Format (gif) y Bitmap (bmp). Un ejemplo de imagen inicial se muestra en la Fig. 2:

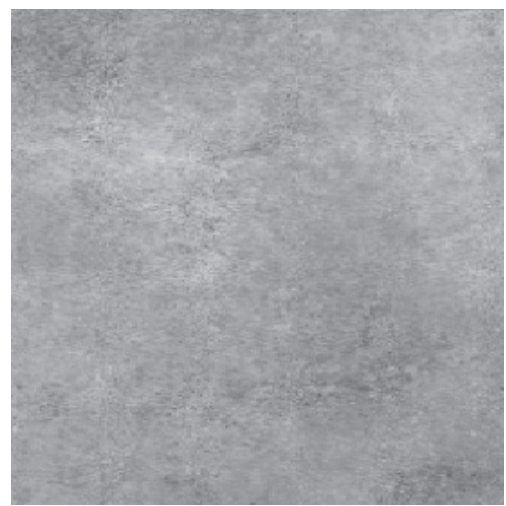

Fig. 2. Ejemplo de imagen inicial

Después de seleccionar la imagen inicial, se aplica un algoritmo para construir el fondo de la imagen de salida o imagen final. El fondo puede considerarse como un resultado preliminar de la propia imagen de salida, pero sin depurar. El tamaño y la resolución del fondo son conocidos, puesto que deben coincidir con el tamaño y la resolución de la imagen final, y por tanto son requerimientos de diseño. El fondo es construido primero como una imagen vacía (matriz nula o matriz de ceros), con el tamaño y resolución especificados.

A continuación, este fondo es rellenado con repeticiones simétricas de la imagen inicial. Este tipo de repetición se realiza mediante un algoritmo conocido como "mirrorshaped" (forma de espejo). El algoritmo "mirror-shaped" se emplea a menudo para aumentar el tamaño de una imagen, manteniendo la continuidad de algunas de sus texturas y preservando la resolución de la imagen final. Un mosaico de la imagen inicial podría también haber sido usado para crear el fondo, pero el resultado ofrecido por el algoritmo de espejo se aproxima más al resultado deseado para la imagen final, por lo que menos procesamiento será necesario después de construir el fondo.

El tamaño de la imagen inicial debe ser determinado, de tal forma que sea posible establecer el número de repeticiones en forma de espejo que se deben hacer en las dos direcciones planas x e y para obtener la imagen final. Por ejemplo, si la imagen de entrada mide $60 \times 60 \mathrm{~cm}$, y la imagen de salida debe medir $1.2 \times 12 \mathrm{~m}$, la imagen de entrada debe ser repetida 2 veces en altura y 20 en longitud sobre el fondo. Las sucesivas simetrías tiene lugar una a una, es decir, cada par

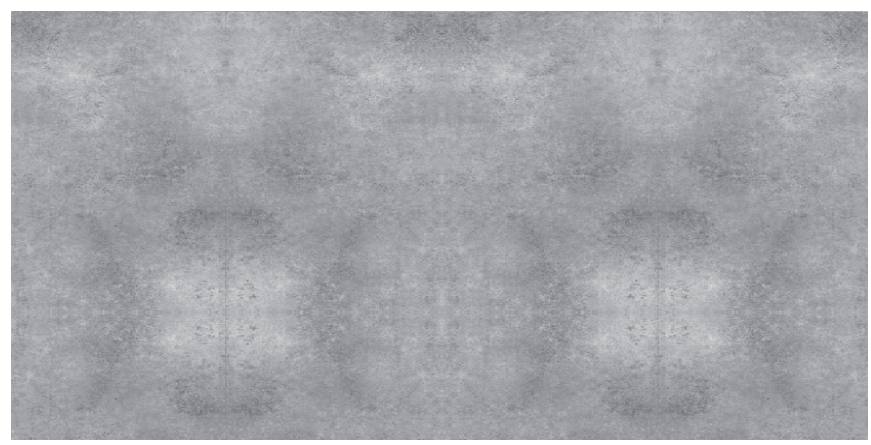

Fig. 3. Imagen de fondo de imágenes simétricas se convierten en la nueva imagen de la siguiente simetría. El proceso continua hasta que el fondo está totalmente cubierto por las simetrías. La Fig. 3 muestra el fondo obtenido tras el procesamiento comentado, a partir de la imagen mostrada en la Fig. 2.

Como se ha indicado previamente, el fondo puede ser considerado una primera aproximación a la solución del problema de la ampliación homogénea de la imagen inicial. Sin embargo, el fondo debe continuar siendo tratado, puesto que los límites de las diferentes réplicas pueden ser apreciados, así como otras trazas dejadas al realizar las distintas simetrías.

Precisamente con el objetivo de eliminar esas trazas de simetría, se ha desarrollado el siguiente proceso. Una librería con diez patrones de textura cerámica fue definida en base a la experiencia de los diseñadores cerámicos que han colaborado en la esta investigación. Estos expertos revisaron el catálogo actual de su empresa, y también los catálogos de recientes temporadas pasadas, y determinaron los diez patrones más habituales. Diez puede parecer un número reducido de patrones, pero la realidad es que los diseños cerámicos basados en texturas aleatorias se pueden aglutinar en unas pocas variantes realmente diferentes.

Cada patrón representa una textura habitual dentro de las superficies cerámicas decoradas y está formado por figuras aleatorias de naturaleza similar. Los patrones se crearon en blanco y negro, de forma que sus contornos pudieran ser detectados y manipulados fácilmente con el procesamiento de imagen posterior. Los patrones fueron creados con una herramienta comercial de dibujo digital. La Fig. 4 muestra un ejemplo de uno de estos patrones.

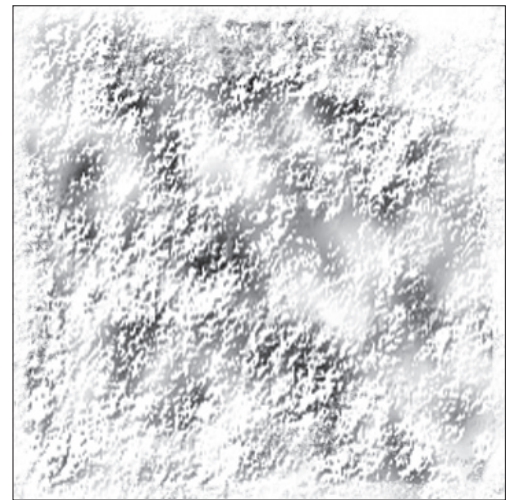

Fig.4. Ejemplo de patrón.

Para el tratamiento de una imagen en particular, un grupo de patrones de la librería es seleccionado por el diseñador. Cada uno de los patrones seleccionados se superpone cíclicamente a la imagen inicial, hasta que la imagen se cubre completamente. Típicamente, los patrones son del orden de ocho veces inferiores a la imagen inicial, por lo que una media de ocho superposiciones son necesarias para cubrir la imagen inicial. En cada superposición se ejecuta un algoritmo que encuentra los píxeles en común entre la imagen de entrada y el patrón. Las coordenadas de color (en el espacio CMY) de los puntos coincidentes se almacenan en matrices temporales tras cada superposición, en la celda correspondiente a la posición del píxel común, tomando como referencia la imagen patrón. Las celdas de las matrices que representan píxeles no coincidentes permanecen en blanco. 
Finalmente, las matrices temporales son integradas en la imagen de fondo. La integración de la información contenida en cada matriz temporal se realiza en un área aleatoria del fondo. Con esto se trata de evitar cualquier coincidencia con las coordenadas originales de la imagen inicial, ya sea en la propia imagen inicial, o en las sucesivas réplicas creadas para el fondo. Dado que las matrices temporales sólo contienen información de los píxeles coincidentes, cualquier otro píxel no sufre ninguna alteración, y permanece tal y como se hubiera definido para el fondo. La Fig. 5 muestra el fondo presentado en la Fig. 3, tras el nuevo procesamiento.

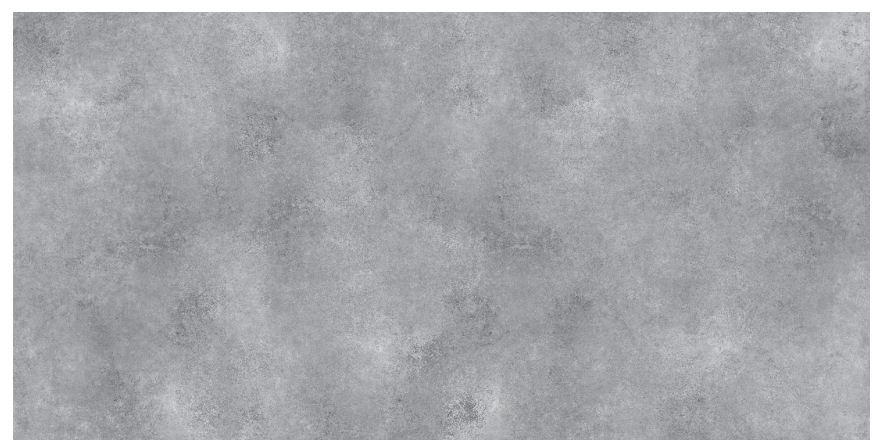

Fig.5. Imagen final.

La imagen final se convierte en la imagen de entrada del sistema de decoración inkjet. Este sistema es capaz de tomar las baldosas sin decorar necesarias, para imprimir sobre ellas la imagen completa. El resultado es un mosaico de baldosas cerámicas, listas para cubrir el espacio deseado con la imagen completa. Una presentación de este mosaico, derivado de la imagen mostrada en la Fig. 5, se presenta en la Fig. 6.

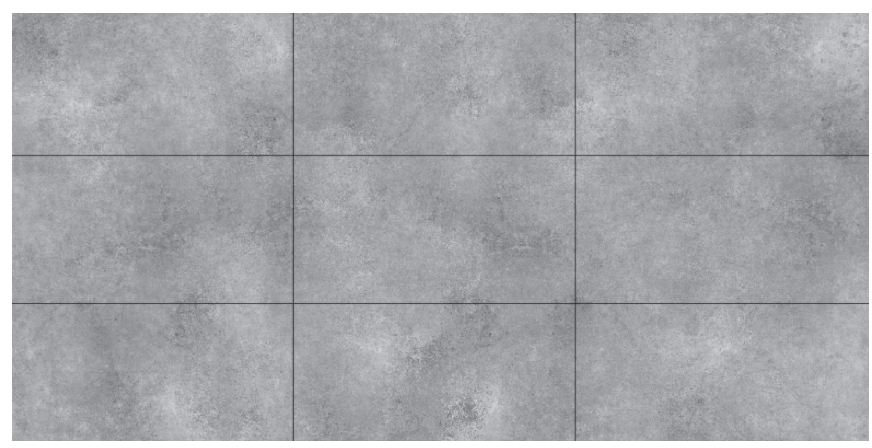

Fig. 6. Lote de baldosas imprimidas con la imagen final mediante inyección de tinta.

La continuidad de los diferentes rasgos geométricos y de color del lote completo, una vez instalado en el suelo, se garantiza siempre que las baldosas se coloquen reproduciendo la imagen final.

\section{DISCUSIÓN}

Es necesario tener en cuenta que el tipo y cantidad de patrones elegidos en el procesamiento determinan la apariencia de la imagen final, así como el método aleatorio de incrustación de las matrices temporales en el fondo. Por lo tanto, el diseñador cerámico puede investigar distintas combinaciones, todas ellas derivadas de la misma imagen inicial, y generar distintas imágenes finales y por tanto distintos modelos cerámicos, y seleccionar aquel que mejor se ajuste a sus necesidades.

La oportunidad de generar un rango tan amplio de modelos cerámicos en un tiempo reducido, es muy aconsejable cuando se pretende ampliar el espectro de modelos del departamento de diseño de una empresa productora de baldosa cerámica. Como se ha comentado en este trabajo, la flexibilidad es un factor clave de competitividad en la industria cerámica actual.

Por tanto, el interés de la herramienta presentada radica en la automatización tanto de la creación de la imagen de gran formato de la textura homogénea, como en su refinamiento alrededor de las áreas susceptibles de perder homogeneidad. El diseñador puede centrarse en seleccionar las mejores imágenes iniciales y combinar los distintos patrones del programa de formas diversas, incluso incluir nuevos patrones, para obtener resultados diferentes. Todos los resultados darán lugar a imágenes finales de texturas homogéneas de las características deseadas, pero con apariencias diferentes, de tal manera que el diseñador podrá optar por el modelo o modelos que considere oportunos en cada ocasión.

\section{RESULTADOS}

El programa informático prototipo desarrollado ha sido probado por el departamento de diseño de la empresa de pigmentos y esmaltes cerámicos Smalticeram S.A. Tras las pruebas se ha podido comprobar que las imágenes finales generadas mediante diseño asistido tenían la misma calidad que las imágenes finales generadas íntegramente por el diseñador sin asistencia. En relación al tiempo empleado en cada caso, la asistencia proporcionó un ahorro medio del orden del $20 \%$ del tiempo de diseño. Incluso en los casos más complejos, en los que fue necesario aplicar un postprocesado manual también asistido, el tiempo empleado por el mismo diseñador para adaptar una imagen a impresión inkjet disminuyó hasta $15 \%$ aproximadamente.

Esta disminución del tiempo de diseño supone un ahorro económico relevante, ya que el acorte del tiempo de diseño reduce el tiempo de desarrollo total del producto. Además, se pueden evaluar un mayor número de decoraciones para satisfacer la demanda del mercado, lo cual eleva la competitividad de la empresa en el sector y puede mejorar su nivel de ventas.

\section{AGRADECIMIENTOS}

Este trabajo ha sido financiado por la Empresa Smalticeram S.A. dentro del proyecto "JUST TO PRINT" SEI-010000-2009-453 financiado por el Ministerio de Industria Turismo y Comercio; y por la Universidad Politécnica de Valencia en el marco del proyecto “DESARROLLO DE TÉCNICAS DE PROCESADO DE IMAGEN DIGITAL PARA LA CREACIÓN DE DECORACIÓN CERÁMICA FLEXIBLE" dentro de Programa de Apoyo a la Investigación y Desarrollo U.P.V. (PAID-05-10).

\section{REFERENCIAS}

1. A. Low, "Introductory Computer Vision and Image Processing", PrenticeHall, Englewood Cliffs, New Jersey, USA, 1989. 
2. Colores Cerámicos S.A. y Talleres Foro S.L., "Decoración digital sostenible de productos cerámicos mediante chorro de tinta continuo y tintas en base agua", Bol. Soc. Esp. Cerám. Vidrio, 49(2), 139-141, 2010.

3. Ferro Spain S.A. ,"Tintas Ink-Jet para Decoración 3D" Bol. Soc. Esp. Cerám. Vidrio, 50(2), XXVII-XXX, marzo-abril, 2011.

4. Esmalglass-Itaca Grupo S.A., "Esmaltes Digitales para un proceso de esmaltación y decoración totalmente digital", Bol. Soc. Esp. Cerám. Vidrio, 50(2), XXIII-XXVI, marzo-abril 2011.

5. J. Gálvez, D. Gálvez, “Mejoras prácticas para líneas de decoración Inkjet en cerámica. Máquina IR de radiaciones electromagnéticas (tecnología de longitud de onda)", Bol. Soc. Esp. Cerám. Vidrio, 51(2), XVII-XX, MarzoAbril, 2012.

6. Ferro Spain S.A., "Desarrollo de efectos cerámicos como acabados superficiales, mediante tecnología de inyección digital", Bol. Soc. Esp. Cerám. Vidrio, 51(2), XLVII-L, Marzo-Abril, 2012.

7. H. Elbehiery, A. Hefnawy, and M. Elewa, "Surface Defects Detection for Ceramic Tiles Using Image Processing and Morphological Techniques", Proceedings of World Academy of Science, Engineering and Technology, 5, 158-160, 2005.

8. C. Boukouvalas, J. Kittler, R. Marik, M. Mirmehdiand, M. Petrou, “Ceramic Tile Inspection for Colour and Structural Defects", under BRITE-EURAM, project no. BE5638, pp 6, University of Surrey, 2006.

9. Se Ho Choi, Jong Pil Yun, Boyeul Seo, Young Su Park, Sang Woo Kim, "Real-Time Defects Detection Algorithm for High-Speed Steel Bar in Coil", Proceedings of World Academy of Science, Engineering and Technology, 21, 2007.

10. G. M. Atiqur Rahaman, Md. Mobarak Hossain, "Automatic defect detection and classification technique from image: a special case using ceramic tiles" International Journal of Computer Science and Information Security, 1(1), 22-30, 2009.

11. S Kukkonen, H Kälviäinen, J Parkkinen, Color features for quality control in ceramic tile industry, Optical engineering, 40(2), 170- 177, 2001.
12. L Iovine, S Westland, TLV Cheung, "Application of Neugebauer-Based Models to Ceramic Printing", IS\&T / SID Twelfth Color Imaging Conference, 176-180, 2001

13. F. Bondioli et al., "Color matching algorithms in ceramic tile production", Journal of the European Ceramic Society 26(3), 311-316, 2006

14. G. Peris-Fajarnés, Latorre, B. Defez, I. Tortajada,F. Brusola," "Evaluation of color prediction models in the decoration of ceramic tiles" Journal of the Ceramic Society of Japan, 116(1), 146-152, 2008

15. J.M Valiente, M.C Carretero, J.M Gómis, F Albert, "Image Processing Tool for the Purpose of Textile Fabric Modelling", XII ADM International conference on design tools and methods in industrial engineering, pp. G1-56-64, 2001.

16. M Valor, F Albert, J.M Gomis, M Contero, “Textile and Tile Pattern Design Automatic Cataloguing Using Detection of the Plane Symmetry Group", Computer Graphics International, proceedings 112-119, 2003.

17. F Albert, J.M Gomis, J.M Valiente, "Reconstruction techniques in the image analysis of Islamic mosaics from the Alhambra", Computer Graphics International, proceedings 618-621, 2004

18. B. Jähne, "Digital Image Processing, Concepts, Algorithms and Scientific Applications", 4th Edition, Berlin, Alemania, Springer, 1997.

19. Jain A. K. Fundamentals of digital image processing, Prentice-Hall, Englewood Cliffs, New Jersey, EEUU, 1989.

20. C. González, R.E. Woods, "Digital Image Processing", Addison Wesley, Liverpool, Reino Unido, 1993.

21. Jensen J. R. Introductory digital image processing, Prentice-Hall, Englewood Cliffs, New Jersey, EEUU, 1996.

Recibido: 25/06/2012

Recibida versión corregida: 24/04/2013

Aceptado: 13/05/2013 